\title{
Response to letter to Editor by: Josef Finsterer
}

\author{
Majid Nejati ${ }^{1}$
}

Accepted: 19 October 2020 / Published online: 5 January 2021

๑) Springer Science+Business Media, LLC, part of Springer Nature 2021

\section{Dear Editor of Heart Failure Reviews}

We would like to thank the authors of the letter for their interest in our paper and for taking the time to express their concerns. Herein, we reviewed their letter about our published review article in Heart Failure Reviews titled, Cardiac manifestations of mitochondrial diseases, and we point out a few points about it.

In this article, we review some of the cardiac complications of mitochondrial dysfunction. We mentioned some of the mitochondrial and nuclear genes that are involved in MID [1]. Here, we did not intend to state a list of all complications and all of the affected genes.

According to authors' comment, histiocytoid cardiomyopathy (CMP) has been mentioned in this review (Histiocytoid CMP is characterized by the pathogenic histiocyte-like cells within sub endocardium and aggregation of structurally abnormal mitochondria). Moreover, as other CMPs, it could also be manifested by arrhythmia and sudden cardiac death [2]

Here, we cited atrial fibrillation (AF) as a cause of stroke. $\mathrm{AF}$ treatment requires in-depth evaluation of the CHA2DS2VASc score as a guiding strategy. However, we did not focus on $\mathrm{AF}$ treatment.

Also, autonomic nervous dysfunction has been listed in Table 2 as a cardiac abnormality in MID.

Heteroplasmy rate was also reported in this review (heteroplasmy level, as a biomarker of MID, is a predisposing factor for atherosclerosis, coronary artery disease, and myocardial infarction). Heteroplasmy rate strongly affect the phenotype of MID, but the role of heteroplasmy rate in cardiac pathology is debatable [3, 4].

The association between arterial and pulmonary hypertension with MID and role of cardiac magnetic resonance imaging in diagnosis of MD have also mentioned in this review briefly.

Overall, we reviewed the genetics of MIDs and noted the importance of screening for heart disease as a manifestation of MIDs. However, we are happy to find any information that complements this review.

Best regards.

Majid Nejati

\section{References}

1. Behjati M, Sabri MR, Far ME, Nejati M (2020) Cardiac complications in inherited mitochondrial diseases. Heart Fail Rev, 1-13.

2. Xie, H., Chen, X., Chen, N., \& Zhou, Q. (2017). Sudden death in a male infant due to histiocytoid cardiomyopathy: an autopsy case and review of theliterature. Am J Forensic Med Pathol, 38(1), 32-34.

3. Perales-Clemente, E., Cook, A. N., Evans, J. M., Roellinger, S., Secreto, F., Emmanuele, V. Schon, E. A. (2016). Natural underlying mt DNA heteroplasmy as a potential source of intra-person hi PSC variability. EMBO J, 35(18), 1979-1990.

4. Takeda, A., Murayama, K., Okazaki, Y., Imai-Okazaki, A., Ohtake, A., Takakuwa, E., Nagai, A. (2020). Advanced pathological study for definite diagnosis of mitochondrial cardiomyopathy. $J$ Clin Pathol.

Publisher's Note Springer Nature remains neutral with regard to jurisdictional claims in published maps and institutional affiliations.
Majid Nejati

mnejatimt@gmail.com

1 Anatomical Sciences Research, Kashan University of Medical Sciences, Qotb Ravandi Blv., 8715981151 Kashan, Islamic Republic of Iran, Iran 